\title{
Effective-periodicity effects in Fibonacci slot arrays
}

\author{
J. K. Hamilton $\odot,,^{1,2, *}$ M. Camacho $\odot,{ }^{3, \dagger}$ R. R. Boix $\odot,{ }^{3}$ I. R. Hooper $\odot,{ }^{2}$ and C. R. Lawrence ${ }^{1}$ \\ ${ }^{1}$ Applied Science, QinetiQ Ltd., Cody Technology Park, Farnborough GU14 OLX, United Kingdom \\ ${ }^{2}$ Department of Physics and Astronomy, University of Exeter, Exeter, Devon EX4 4QL, United Kingdom \\ ${ }^{3}$ Department of Electronics and Electromagnetism, Universidad de Sevilla, 41012 Seville, Spain
}

(Received 20 August 2021; revised 16 December 2021; accepted 16 December 2021; published 29 December 2021)

\begin{abstract}
In this Letter, the transmission properties of a nonperiodic array of slots arranged in the form of a Fibonacci sequence are investigated. By arranging the slots in this manner, an additional periodicity can be utilized, resulting in corresponding resonance features in the transmitted signal. By investigating the transmission response of a perforated metallic sheet over a broad frequency range $(6-40 \mathrm{GHz})$, it is shown that this simple one-dimensional chain supports two periodicities, one due to the regular periodic separation and one due to average spacing-which is related to the golden ratio. This response replicates the resonant behavior of a two-dimensional periodic array with a single nonperiodic array also creating new families of diffraction lobes in the far-field region.
\end{abstract}

DOI: 10.1103/PhysRevB.104.L241412

Since the discovery of extraordinary optical transmission (EOT) through periodically perforated metallic sheets [1] there has been a focus on understanding and controlling the transmission resonances in such structures [2]. The finding that the resonant nature of the transmission through periodic arrays of apertures was due not only to the aperture's geometry but mostly to their arrangement opened a number of opportunities for the development of the later field of metasurfaces and metamaterials $[3,4]$. A vast number of theoretical and experimental studies have been focused on the transmission resonances of periodic arrangements of subwavelength apertures cut into metallic sheets, including: one-dimensional slit and holes arrays [5], two-dimensional hole arrays [6], and single apertures [7].

Despite the large interest in periodic structures, there has only been a limited interest in nonperiodic arrangements of scatterers in the past. However, they have recently attracted considerable attention due to possible additional control over the electromagnetic properties. Nonperiodic or quasiperiodic patterns can result in additional degrees of control of the wave propagation-compared to the periodic case $[8,9]$. One of the most common and well-known nonperiodic structures is the Fibonacci sequence, which has been used as the basis of a variety of previously investigated structures. These include: metamaterial absorbers [10], dielectric multilayers [11,12], slit arrays [13,14], and resonators/antennas [15-17]. There has also been a strong interest in Fibonacci sequences in photonic crystal structures [18-20].

An important distinction that separates random from regular periodic arrays is the nonarithmetic sequence of constructive/destructive features in their transmission spectra [8]. In periodic arrays, they originate from the arithmetic sequence that is followed by the onset of the diffraction modes

\footnotetext{
*jkhamilton@qinetiq.com

†mcamachoa@us.es
}

associated with each periodicity. It has been shown that, in some cases, the appearance of these grazing components introduces energy redistributions responsible for transmission zeros, which are also the origin of EOT phenomena in the absence of surface plasmons [21]. These grazing wave components have shown a great potential for the excitation of surface lattice resonances when coupled to plasmonic media [22]. As we will also show in the following, these energy redistributions in nonperiodic arrays can also be associated with pseudograting lobes, which can be tracked in frequency in the form a maximum in the radiation diagram.

With the design of metasurfaces being based on transformation optics, it is important for antenna designers to be able to model the behavior of large arrays to validate their designs. In parallel, the optics community has put a considerable effort into the characterization of quasicrystal arrangements as they present short-range disorder whereas maintaining long-range order, leading to well-defined diffractive behavior with the potential for novel multiresonant phenomena. From a computational perspective, they are a challenge as they require the analysis of hundreds of coupled elements with very few symmetries, if any. Let us focus, for instance, on the case of one-dimensional Fibonacci chains of slots in a metal sheet.

The Fibonacci sequence is defined such that each number is the sum of the two preceding ones (for example: $F_{n}=$ $\left.F_{n-1}+F_{n-2}\right)$. One key feature of the Fibonacci sequence is that when we take any two successive Fibonacci numbers, their ratio tends to the golden ratio as $n$ increases.

The golden ratio $\varphi$ is an irrational number that is a solution of the quadratic equation,

$$
\varphi^{2}-\varphi-1=0,
$$

given by

$$
\varphi=\frac{1+\sqrt{5}}{2} \approx 1.618
$$




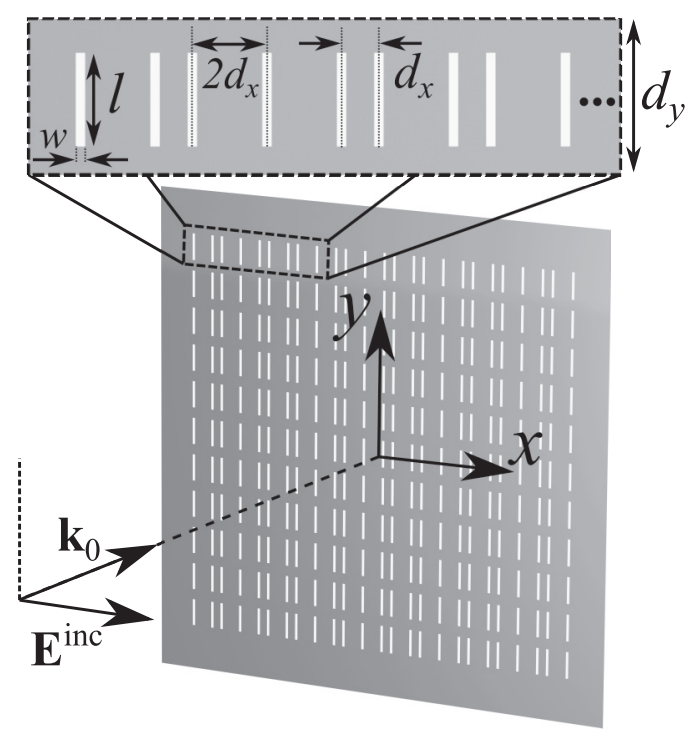

FIG. 1. Schematic showing the experimental sample: a perforated metallic sheet, depicting the slot separation $d_{x}$ and slot width $w$. The periodicity along $y$ is chosen such that it does not contribute to the diffractive behavior whereas increasing the transmitted signal.

To create one-dimensional Fibonacci chains of slots, let us define $A$ and $B$ as metal spacing and slot, respectively. The Fibonacci sequence of spacings and slots is achieved at each generation step by using the recurrent substitution given by

$$
\begin{aligned}
& A \rightarrow A B, \\
& B \rightarrow A,
\end{aligned}
$$

which is initialized by an element $A$ [23]. Further iterations are created by concatenating the previous two iterations in the sequence, for example: $A B+A=A B A$. This is further shown by the first five iterations,

$$
\begin{gathered}
A B, \\
A B A, \\
A B A A B, \\
A B A A B A B A, \\
A B A A B A B A A B A A B .
\end{gathered}
$$

From this sequence, we can generate our array of slots by identifying each $A$ with a metal spacing and each $B$ with a slot. The iterations above show that there are two possible centerto-center slot separations $A$ and $A A$. For the case of notation, these two slot separations will be represented as $d_{x}$ and $2 d_{x}$, and the slot width will be represented by $w$.

A finite chain of 30 slots was created by perforating a metallic sheet with slot width $w=0.25 d_{x}$ and slot length $l_{s}=$ $2.4 d_{x}$. Figure 1 shows a schematic of the idealized perforated metallic sheet. To maximize the transmission signal through the sample, additional rows of slots were added, although the coupling between adjacent rows is negligible due to the symmetry of the slots and the chosen [24]. Therefore, the associated reciprocal space vector in the $y$ direction does not play any role at normal incidence. The value of $d_{x}$ was chosen to be $20 \mathrm{~mm}$ with a slot width $w$ of $5 \mathrm{~mm}$, slot length $l$ of $48 \mathrm{~mm}$, and a center-to-center vertical separation $d_{y}$ of $68 \mathrm{~mm}$.

If one studies the average spacing between the elements in the array in the limit of small slot widths, one finds that it corresponds to $d_{x} \varphi$, where $\varphi$ is the golden ratio. This can be observed when taking a ratio between the number of slots and the total number of $A \mathrm{~s}$ in a sequence. For example, 34 slots correspond to $55 \mathrm{As}$, resulting in a ratio of 1.618. As we will demonstrate below, it can been found that the position of the features can be predicted by assuming that the array presents two periodicities: one associated with the spacing $d_{x}$ and one associated with the average spacing $d_{x} \varphi$ such that each of those can be represented by reciprocal space vectors,

$$
k_{m}=\frac{2 \pi m}{d_{x}}, \quad k_{n}=\frac{2 \pi n}{d_{x} \varphi},
$$

with $m$ and $n$ being integer numbers. Then one could expect a feature at frequencies for which $k_{0}=k_{m}+k_{n}$ for all values of $m$ and $n$, although, in practice, we will expect their coupling to free space radiation to decrease with their order. This behavior allows one to replicate the resonant behavior of a two-dimensional periodic array with a single nonperiodic array.

From Eq. (3), it appears that the Fibonacci design may give some advantages over the standard periodic arrays; namely, additional resonances due to effective periodicities. To demonstrate the above behavior, and to further understand these nonperiodic structures, a numerical method of moments (MoM) code was utilized.

The analysis of large nonperiodic arrays of scatterers is a complex computational problem due to the large electrical size (i.e., in terms of the wavelength). However, surface integral equation methods have been developed that allow for the solution of scattering problems from the discretization of the surface using analytical expansions of the fields on either side [25]. In particular, for the system of interest here the method of moments reduces the problem to finding the electric-field distribution on the surface of each of the $M$ slots that can be shown to obey the following integral equation:

$$
\begin{aligned}
& \mathbf{J}^{\mathrm{as}}(x, y)+\sum_{j=1}^{M} \iint_{\eta_{j}} \overline{\mathbf{G}}_{M}\left(x-x^{\prime}, y-y^{\prime}\right) \\
& \mathbf{E}_{t}^{s c}\left(x^{\prime}, y^{\prime}, z=0\right) d x^{\prime} d y^{\prime}=\mathbf{0} \quad(x, y) \in \eta_{i} \\
& (i=1, \ldots, M),
\end{aligned}
$$

where $\mathbf{J}^{\text {as }}(x, y)$ is the surface current in the absence of the slots (i.e., generated by the incident illumination on an infinite perfectly conducting screen), $\overline{\mathbf{G}}_{M}(x, y)$ is the dyadic Green's function that relates the tangential electric field at the origin with the electric current density at a given point and $\eta_{i}$ is the surface of the $i$ th slot.

The MoM can be then applied by proposing a set of $N_{b}$ basis functions,

$$
\mathbf{E}_{t}^{s c}(x, y, z=0) \approx \sum_{l=1}^{N_{b}} e_{j l} \mathbf{d}_{j l}(x, y) \quad(x, y) \in \eta_{j},
$$


which can also be used as testing functions to convert (4) into a system of linear equations for the unknown weights $e_{j l}$,

$$
\sum_{j=1}^{M} \sum_{l=1}^{N_{b}} \Delta_{i j}^{k l} e_{j l}=p_{i k} \quad\left(i=1, \ldots, M ; k=1, \ldots, N_{b}\right),
$$

where

$$
\begin{aligned}
\Delta_{i j}^{k l}= & \iint_{\eta_{i}} \mathbf{d}_{i k}^{*}(x, y)\left[\iint_{\eta_{j}} \overline{\mathbf{G}}_{M}\left(x-x^{\prime}, y-y^{\prime}\right)\right. \\
& \left.\times \mathbf{d}_{j l}\left(x^{\prime}, y^{\prime}\right) d x^{\prime} d y^{\prime}\right] d x d y \\
& \times\left(i, j=1, \ldots, M ; k, l=1, \ldots, N_{b}\right),
\end{aligned}
$$

and where

$$
\begin{aligned}
p_{i k}= & -\left(\iint_{\eta_{i}} \mathbf{d}_{i k}^{*}(x, y) \mathbf{J}^{\mathrm{as}}(x, y) d x d y\right) \\
& \times\left(i=1, \ldots, M ; k=1, \ldots, N_{b}\right) .
\end{aligned}
$$

Details on how to efficiently calculate the matrix elements in (7) can be found elsewhere [25]. Once this system is solved, the electric field on each of the slots can be obtained using (5) as well as its Fourier transform and, in turn, the transmitted power in a straightforward manner [26].

To demonstrate the effect of arranging our slots following the Fibonacci sequence, we have calculated the transmission response of three different one-dimensional slot arrays, two periodic arrays, one with period $d_{x}$ and one with $d_{x} \varphi$, and a Fibonacci array. Due to the frequency associated with the onset of the $n=1$ diffracted order of an array with $d_{x}=$ 20-mm periodicity being $15 \mathrm{GHz}$, the transmission response was studied over a broad frequency range of $5.8-40 \mathrm{GHz}$ with the polarization perpendicular to the slots' axis. The parallel polarization was not investigated due to the slots not being resonant in this orientation - in the array acting as a solid metallic sheet for the frequency range of interest. Figure 2 shows the response for the three structures as well as the predicted modes for the Fibonacci array-using the expressions in (3) which coincide with well-defined peaks of the Fourier spectrum of the Fibonacci array. For ease of comparison, the transmitted power is normalized to the maximum value.

For the case with constant period $d_{x}$, there are clear and strong resonances at 15 and $30 \mathrm{GHz}$ : These are the fundamental resonances of the slots with period $d_{x}$ associated with the onset of diffraction modes. When the period is increased to $d_{x} \varphi$, the fundamental resonances shift down in frequency to 9.2 and $18.5 \mathrm{GHz}$, respectively. For the $d_{x} \varphi$ case, additional higher-order modes (27.9 and $37.1 \mathrm{GHz}$ ) are visible due to the longer period. The predicted features-using Eq. (3) - for the Fibonacci array have been represented using dotted lines in Fig. 2. To further verify the predicted modes, a fast Fourier transform has been performed on the geometry associated with a one-dimensional Fibonacci chain as can be seen in Fig. 2. This allows us to have an estimate of the coupling strength of the modes with plane-wave illumination; larger Fourier amplitudes would be expected to give rise to stronger coupling.
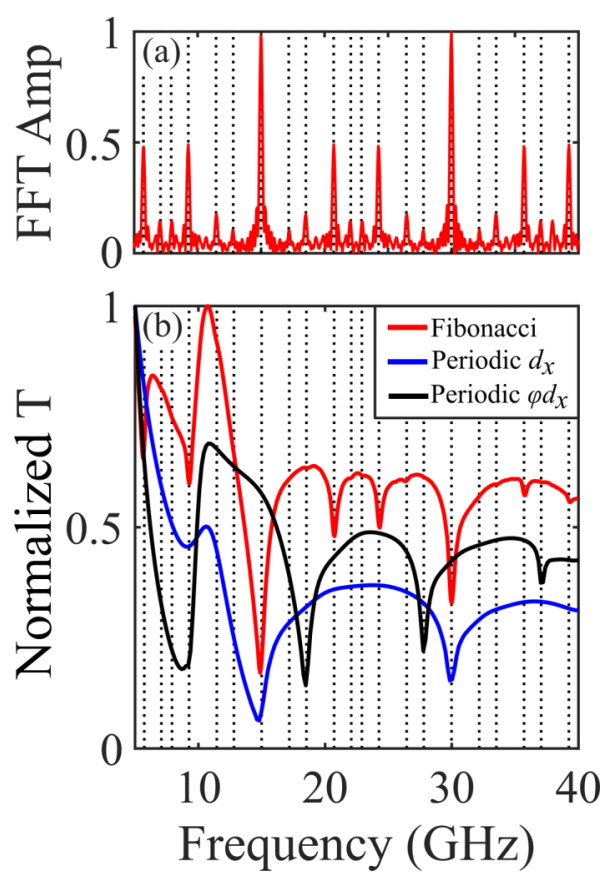

FIG. 2. (a) Normalized Fourier amplitude for a one-dimensional Fibonacci chain. (b) Comparison of the transmission response from a periodic slot array with period $d_{x}$, period $d_{x} \varphi$, and a Fibonacci slot array. The transmission is normalized to the maximum. All three structures contain 30 slots in the array.

For the Fibonacci array, additional modes corresponding to those predicted by Eq. (3) are present. These modes can be observed-most prominently-in Fig. 3(a) at 20.7, 24.3, and $35.7 \mathrm{GHz}$; however, poorly coupled modes are also observed at the other predicted frequencies. All their positions are shown with dotted lines.

Figure 3(a) shows the normalized transmitted power for arrays with increasing slot numbers in the $x$ direction. It is clear that the resonant modes are not introduced by the increasing number of slots. However, we find that the modes become better defined as the number of slots increases, increasing the relative depth and quality factor of the predicted minima. Additional simulations were also run to identify the influence of the slot width [Fig. 3(b)] and showed that this results in a change in the coupling strength to the Fibonacci modes but not to their frequency.

To confirm the results obtained from the simulations, i.e., the presence of additional resonances that lead to abrupt changes in the far-field transmitted power, a sample was built and measured. The slot array was fabricated by laser cutting slots onto a 3-mm-thick copper sheet, whose thickness and conductivity within the frequency range of interest can be accurately approximated by a negligible-thickness perfect electric conductor. The overall sample size was $1 \mathrm{~m}^{2}$ with 30 slots in each row and 14 rows.

The transmission of the sample was measured by using a large-scale focused horn setup with a series of banded horns. The spherical mirrors and horn antennas used for the experiment produced a large beam diameter (resembling plane-wave illumination), therefore, maximizing the illumination area of the sample [27]. To mimic the simulations, the 


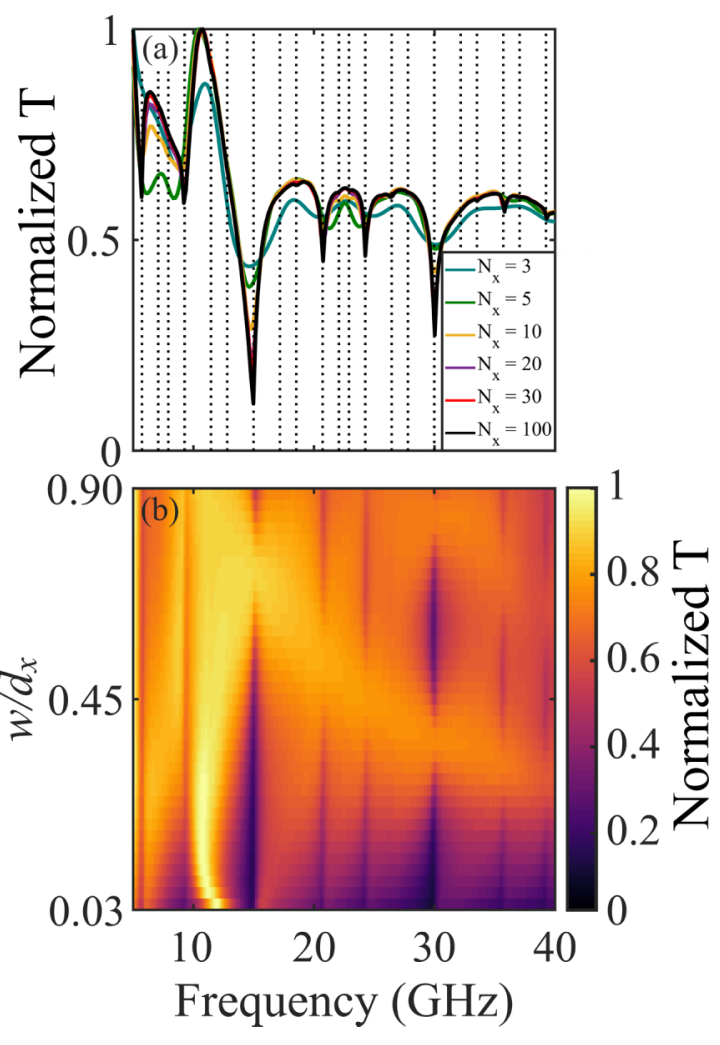

FIG. 3. Calculated transmission response through a Fibonacci slot array of (a) varying slot number $\left(N_{x}\right)$ with parameters; $d_{x}=20$, $w=5 \mathrm{~mm}$, and slot length of $48 \mathrm{~mm}$. (b) Varying the slot width from $0.025 d_{x}$ to $0.900 d_{x}$, whereas keeping the total number of slots as 30 . The transmission is normalized to the maximum value in the range.

sample was illuminated at normal incidencewith the polarization perpendicular to the slots' axis over a frequency range of 5.8-40 GHz.

Figure 4 shows the transmission spectrum under the aforementioned experimental conditions. The blue curve shows the data with a time-gating technique applied to remove unwanted reflections from the testing room. As predicted from the modeling, there are two strong resonances at 15 and $30 \mathrm{GHz}$. These are the fundamental modes that would be present for a periodic array of slots with $d_{x}=20 \mathrm{~mm}$. Additional modes can be seen throughout the investigated frequency range (i.e., 20.7, 24.6, and $26.7 \mathrm{GHz}$ ). The modes that have been found experimentally are shown with gray arrows.

It is rather difficult to find the corresponding feature to all vertical dashed lines shown on previous figures, but it is apparent that some do present a correspondence in the measured spectrum. Since the strength of the modes depends on the coupling to the electric-field distribution on the slot and given the decaying Fourier amplitude with the magnitude of the mode's wave vector, higher-order modes will be more difficult to find experimentally due to the noise. Also, one needs to consider the fact that the experiment was performed in an environment that could not be introduced into the method of moments analysis using a mirror setup which prevents us from getting a perfect and phase-homogeneous plane wave. Due to these factors and for a fair comparison, the MoM-predicted

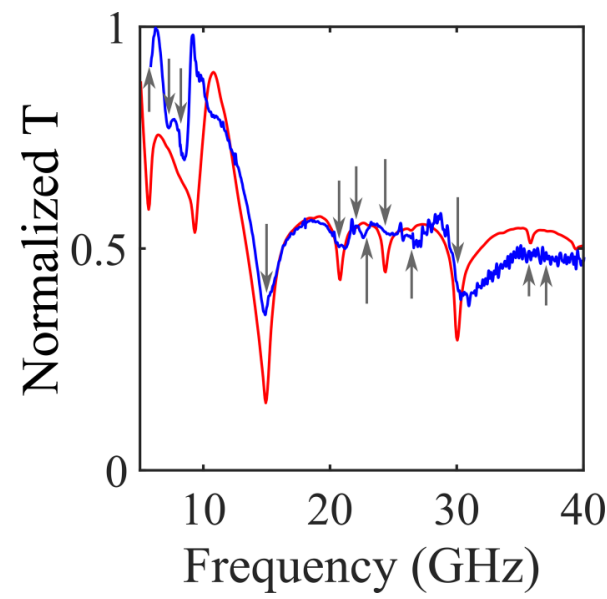

FIG. 4. Transmission through the free standing 30 by 14 slot array sample with parameters; $d_{x}=20, w=5 \mathrm{~mm}$, slot length of $48 \mathrm{~mm}$, and center-to-center vertical separation of $68 \mathrm{~mm}$. The array was illuminated at normal incidence with the polarization perpendicular to the slots. The red curve shows the MoM-predicted transmission that has been scaled down by a factor of 0.9 , and the blue curve shows the experimental data with a time gating applied. The gray arrows indicate the modes predicted by Eq. (3) that coincide with measured features.

transmission shown in Fig. 4 has been scaled down by a factor of 0.9 .

The presence of these resonances can be understood from a far-field perspective as the onset of pseudograting lobes (i.e., directions at which the interference of the fields emitted by each slot add up constructively). To confirm this hypothesis, we have numerically studied the far-field radiation pattern for two sample frequencies-20 and $40 \mathrm{GHz}$ - finding many large-emission peaks on the $x-z$ plane as shown in Fig. 5. By applying simple trigonometrics, one can extract the predicted directions at which the combinations of the $x$-direction wavevectors $k_{m}+k_{n}$ shown in (3) would emit and have marked them on the two figures with dashed lines. For ease of interpretation, we have limited the combinations to $m, n \in[-3,3]$, finding that each combination corresponds to one well-defined far-field emission peak. For comparison, we show on the right the radiation pattern of the equivalent one-dimensional periodic array with the same geometrical parameters for the slots and periodicity of $d_{x}=20 \mathrm{~mm}$ for which one finds three grating lobes $(m=-1,0,1)$ at $20 \mathrm{GHz}$ and four $(m=$ $-2,-1,0,1,2)$ at $40 \mathrm{GHz}$ whose theoretical locations are also marked with dashed lines. For the calculation of this far field, we have used the exact procedure shown elsewhere [26] from the approximate solution of the well-validated method of moments in-house implementation.

It is worth noting that the indices of the Fibonacci wave vectors are not monotonically distributed in the far field as one finds in traditional periodic arrays for which larger indices (higher modes) emit at larger angles which are closer to grazing. Here, because of the combination of the two wave vectors, we find that, at $40 \mathrm{GHz}$, the mode $m, n=(3,-3)$ is located near $25^{\circ}$ which is somewhat close to the $(1,0)$ mode which is found at $22^{\circ}$. Similar behavior is observed with the modes $(3,-2)$ and $(0,3)$ at $41^{\circ}$ and $44^{\circ}$, respectively. 

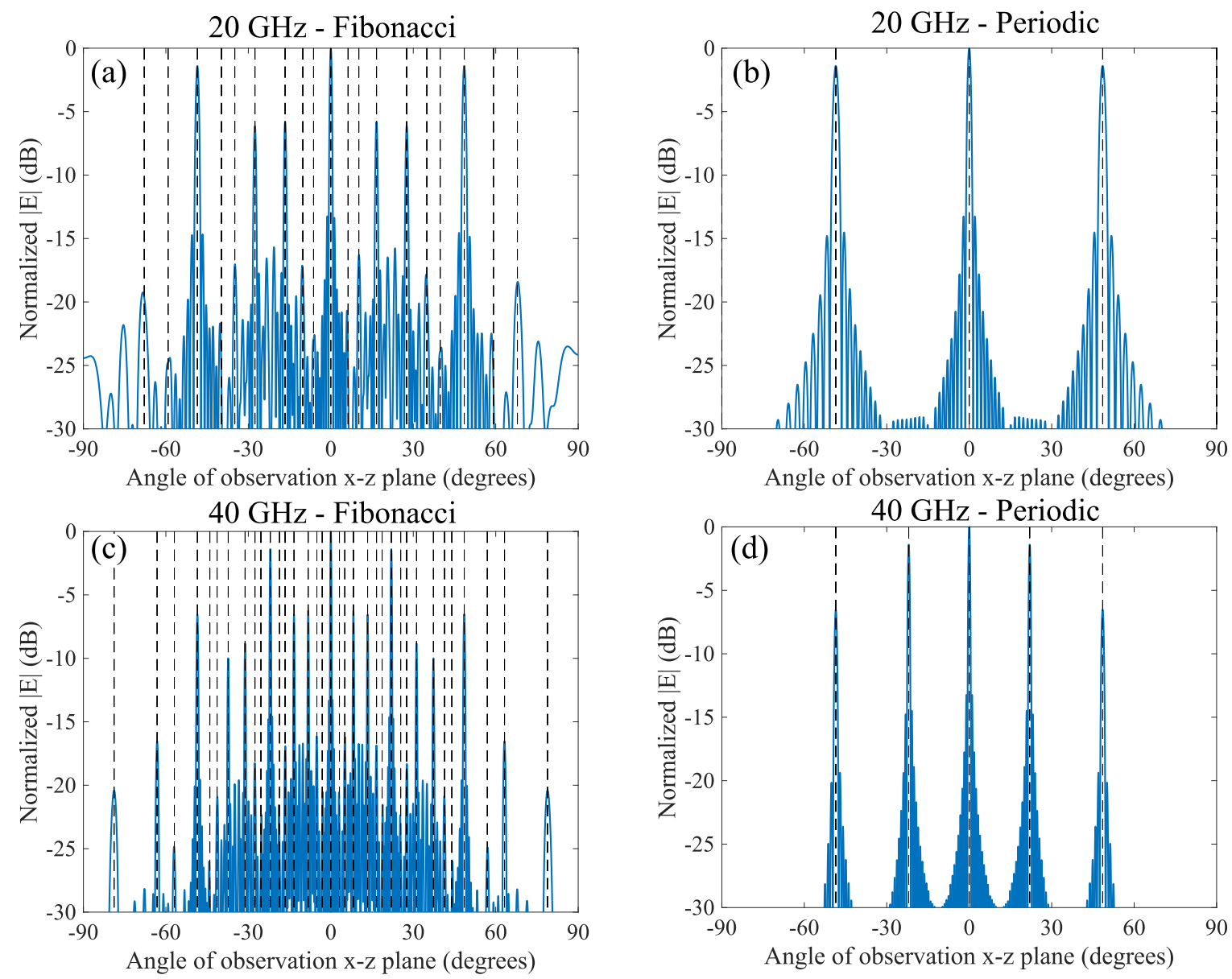

FIG. 5. Angle-resolved far-field spectra of a Fibonacci array of 30 slots and the equivalent periodic array illuminated by a plane wave obtained from the MoM for two different frequencies of 20 and $40 \mathrm{GHz}$. The black dashed lines show the pseudograting lobes predicted from the integer combinations of the in-plane wave vectors in (3).

Among the additional constructive emission directions, we find arguably high electric fields at $20 \mathrm{GHz}$ for the angles $\pm 16.6^{\circ}$ and $\pm 27.6^{\circ}$ with -5.8 and $-6.1 \mathrm{~dB}$, respectively. These correspond to $m, n=( \pm 1, \mp 1)$ and $m, n=(0, \pm 1)$, which do not exist for the equivalent periodic arrays. An additional feature we find is that, although the array is not mirror symmetric with respect to the $y-z$ plane and, correspondingly, the radiation pattern is not symmetric with respect to the zero angle, the radiation maxima follow the symmetry of (3), and each peak associated for any pair $m, n$ is found for $-m,-n$ with the corresponding reversed angle.

This behavior could find applications in multidirectional beam-forming devices, which could explore many more directions than periodic metasurfaces for the same size of the unit cell as well as diffractive surfaces for reduced radar cross section.

To summarize, the transmission properties of a nonperiodic array of slots in a conducting sheet arranged in the form of a Fibonacci sequence have been investigated. It was predicted that additional transmission modes due to an additional effective periodicity would be present, and that these would be re- lated to the golden ratio. We, subsequently, demonstrated the existence of these additional modes both numerically and experimentally. This effective second periodicity allows one to replicate the resonant behavior of a two-dimensional periodic array with a single nonperiodic array. We have also demonstrated that this effect leads to a large number of high-output directions in the radiation diagram of the Fibonacci arrays, whose directions can be predicted from the grating wavevector combinations of the two periodicities of the sequence.

The data that support the findings of this study are available from the corresponding author upon reasonable request.

This work was supported, in part, by the UK Engineering and Physical Sciences Research Council via the Prosperity Partnership, TEAM-A (Grant No. EP/R004781/1) and the Centre for Doctoral Training in Metamaterials (XM2) (Grant No. EP/L015331/1). The work of M. Camacho and R.R.B. has been partially supported by Grant No. PID2020-116739GB-I00 funded by MCIN/AEI/10.13039/501100011033.
[1] T. W. Ebbesen, H. J. Lezec, H. Ghaemi, T. Thio, and P. A. Wolff, Nature (London) 391, 667 (1998).
[2] F. J. García de Abajo, Rev. Mod. Phys. 79, 1267 (2007).

[3] O. Quevedo-Teruel et al., J. Opt. 21, 073002 (2019). 
[4] H. T. Chen, A. J. Taylor, and N. Yu, Rep. Prog. Phys. 79, 076401 (2016).

[5] S. Freer, M. Camacho, S. Kuznetsov, R. Boix, M. Beruete, and M. Navarro-Cía, Photonics Res. 8, 430 (2020).

[6] M. Navarro-Cía, V. Pacheco-Peña, S. A. Kuznetsov, and M. Beruete, Adv. Opt. Mater. 6, 1701312 (2018).

[7] D. R. Jackson, A. A. Oliner, T. Zhao, and J. T. Williams, Radio Sci. 40, RS6S10 (2005).

[8] T. Matsui, A. Agrawal, A. Nahata, and Z. V. Vardeny, Nature (London) 446, 517 (2007).

[9] J. K. Hamilton, I. R. Hooper, and C. R. Lawrence, Adv. Electromagnet. 10, 1 (2021).

[10] Q. Yang, X. Chen, Y. Li, X. Zhang, Y. Xu, Z. Tian, C. Ouyang, J. Gu, J. Han, and W. Zhang, APL Mater. 5, 096107 (2017).

[11] E. Maciá, Appl. Phys. Lett. 73, 3330 (1998).

[12] R. Nava, J. Tagüeña-Martínez, J. Del Rio, and G. Naumis, J. Phys.: Condens. Matter 21, 155901 (2009).

[13] A. Fernández-Domínguez, I. Hernández-Carrasco, L. MartínMoreno, and F. García-Vidal, Electromagnetics 28, 186 (2008).

[14] J. Li, S. Liu, C. Huang, T. Li, Q. Wang, and Y. Zhu, J. Opt. A: Pure Appl. Opt. 10, 075202 (2008).

[15] B. Ozbakis and A. Kustepeli, Microwave Opt. Technol. Lett. 50, 1046 (2008).

[16] C. Sharma and D. K. Vishwakarma, IEEE Antennas Wireless Propagat. Lett. 16, 932 (2017).
[17] M. Chauhan, A. K. Pandey, and B. Mukherjee, Microwave Opt. Technol. Lett. 61, 2268 (2019).

[18] R. B. Capaz, B. Koiller, and S. L. A. de Queiroz, Phys. Rev. B 42, 6402 (1990).

[19] L. Dal Negro, C. J. Oton, Z. Gaburro, L. Pavesi, P. Johnson, A. Lagendijk, R. Righini, M. Colocci, and D. S. Wiersma, Phys. Rev. Lett. 90, 055501 (2003).

[20] M. Tavakoli and Y. S. Jalili, J. Theor. Appl.Phys. 8, 113 (2014).

[21] F. Medina, F. Mesa, and R. Marqués, IEEE Trans. Microwave Theory Tech. 56, 3108 (2008).

[22] V. G. Kravets, A. V. Kabashin, W. L. Barnes, and A. N. Grigorenko, Chem. Rev. 118, 5912 (2018).

[23] M. Ghulinyan, " One-dimensional photonic quasicrystals," in Light Localisation and Lasing: Random and Quasi-random Photonic Structures, edited by M. Ghulinyan and L. Pavesi (Cambridge University Press, U.K., 2014), p. 99-129.

[24] M. Camacho, R. R. Boix, F. Medina, A. P. Hibbins, and J. R. Sambles, Opt. Express 25, 24670 (2017).

[25] M. Camacho, R. R. Boix, and F. Medina, Phys. Rev. E 93, 063312 (2016).

[26] M. Camacho, R. Boix, S. Kuznetsov, M. Beruete, and M. Navarro-Cia, IEEE Trans. Antennas Propag. 67, 6029 (2019).

[27] J. K. Hamilton, S. J. Berry, J. H. Spencer, C. R. Lawrence, F. C. Smith, and T. D. Drysdale, IET Microwaves, Antennas 14, 547 (2020). 